\title{
3d Virtual Histology of Human Cerebellum by Propagation-Based X-Ray Phase- Contrast Tomography
}

Mareike Töpperwien $^{1,2}$, Marina Eckermann ${ }^{1}$, Anna-Lena Robisch ${ }^{1}$, Christine Stadelmann $^{3}$ and Tim Salditt $^{1,2, *}$

1. Institute for X-Ray Physics, University of Göttingen, Göttingen, Germany.

2. Center for Nanoscopy and Molecular Physiology of the Brain, Göttingen, Germany.

3. Institute for Neuropathology, University Medical Center, Göttingen, Germany.

* Corresponding author, tsaldit@gwdg.de

Studies of the human brain cytoarchitecture are routinely performed by classical histology, i.e., by examining the tissue under a light microscope after serial sectioning and subsequent staining. The procedure is labor-intensive and the three-dimensional (3d) anatomy can only be determined after aligning the individual sections, leading to a reconstructed volume at non-isotropic resolution. Additionally, artifacts can be introduced, e.g., by mechanical distortions during the slicing procedure. Propagation-based phase-contrast tomography offers a promising alternative due to the potential resolution and high penetration depth which enables non-destructive imaging of the sample's $3 \mathrm{~d}$ density distribution at high detail.

In this work, we present x-ray phase-contrast tomography of unstained paraffin-embedded human cerebellum performed at our dedicated endstation GINIX installed at the P10 beamline at DESY [1,2]. By exploiting the low divergent illumination of the undulator for imaging and installing a high resolution lens-coupled detector (XSight Micron, Rigaku) in close proximity to the sample, leading to image formation in the direct contrast regime in which phase contrast is visible as edge enhancement, the sample can be imaged at mm-sized field of view and moderate resolution, allowing for the observation of single cells in all layers of the cerebellum (cf. Fig. 1(a-d)). Even sub-cellular details as the dendritic tree as well as nucleus and nucleolus of the large Purkinje cells can be resolved. Note that in this configuration, the resolution of the system is mainly limited by the point-spread function of the detection system.

By inserting a set of Kirkpatrick-Baez mirrors into the beam path as well as placing a waveguide device in its focal plane, acting as a coherence and spatial filter and leading to a secondary source of size well below $20 \mathrm{~nm}[3,4]$, the setup can be changed to a cone-beam geometry in which a higher resolution can be reached due to the small secondary source spot. By replacing the detection system with a fiber-coupled sCMOS camera (Photonic Science) and exploiting a larger propagation distance behind the sample, image formation shifts towards the holographic regime. This allows for high resolution recordings of specific regions of interests, which can be chosen based on the overview scan in parallel-beam geometry, with adjustable geometrical magnification and hence field of view and resolution, down to voxel sizes in the range of a few ten nanometers (cf. Fig. 1(e)). Note that the change of setup can be achieved in only a few minutes so that the sample can principally stay in place during the modifications (not the case for the data shown here). Subsequent automatic cell segmentation based on the spherical Hough transform provides access to the $3 \mathrm{~d}$ cellular distribution within the tissue, enabling extensive statistical analysis based on several thousands of cells [6].

Further, we show that this can be extended to the laboratory by using a liquid-metal jet microfocus source (Excillum $\mathrm{AB}$ ) in an optimized experimental configuration combined with suitable phase retrieval $[5,6]$. 
It provides results with single cell sensitivity in large reconstruction volumes which are consistent with classical histology results and allow for the automatic segmentation of over a million of cells in the densely packed granular layer [7].

\section{References:}

[1] T. Salditt et al, J. Synchrotron Rad. 22 (2015) 867-878.

[2] M. Töpperwien et al, Proc. SPIE 9967 (2016) 99670T.

[3] S.-P. Krüger et al, J, Synchrotron Rad. 19 (2012) 227-236.

[4] S. Hoffmann-Urlaub et al, Microelectron. Eng. 164 (2016) 135-138.

[5] M. Töpperwien et al, Sci. Rep. 7 (2017) 42847

[6] M. Töpperwien et al, PNAS (accepted for publication)

[7] The authors acknowledge funding from the Cluster of Excellence 171 Nanoscale Microscopy and Molecular Physiology of the Brain and the Collaborative Research Center 755 Nanoscale Photonic Imaging of the German science foundation (DFG).

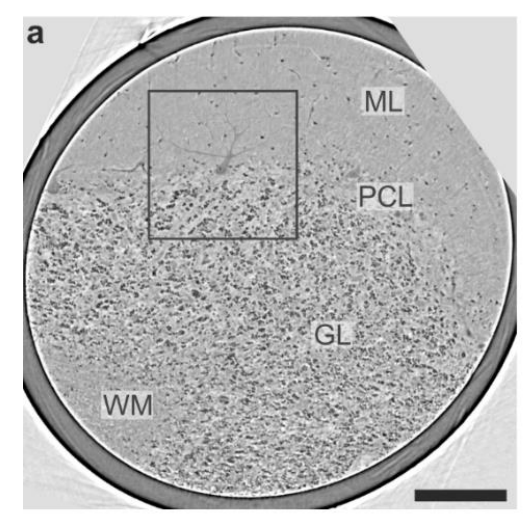

d

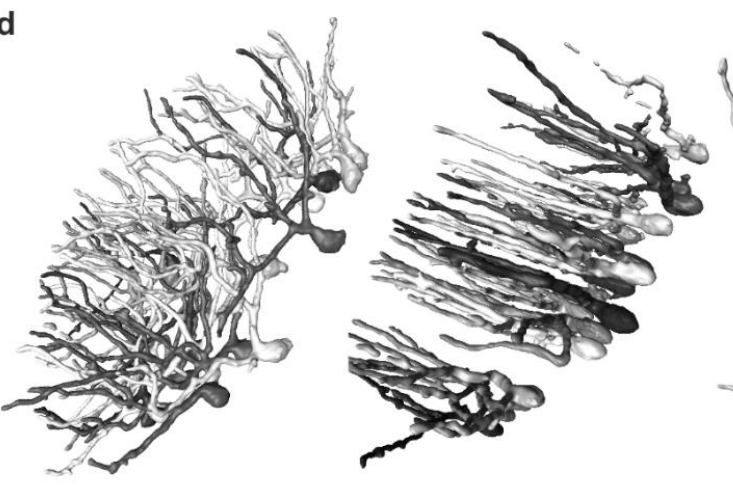

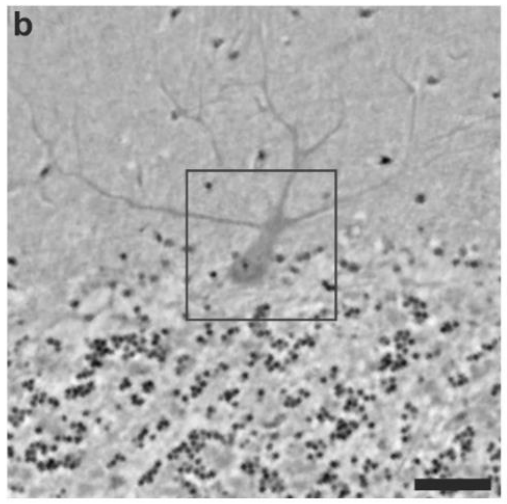
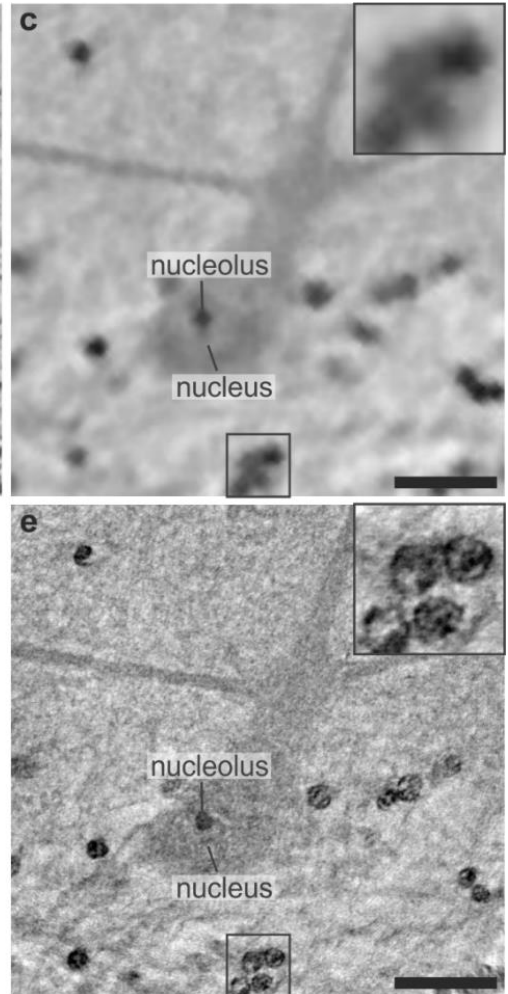

Figure $13 \mathrm{~d}$ visualization of a $1 \mathrm{~mm}$ punch from paraffin-embedded human cerebellum measured at the GINIX setup both in parallel and cone-beam geometry. (a) Virtual slice through the volume obtained in the parallel-beam geometry (pixel size: $550 \mathrm{~nm}$, energy: $13.8 \mathrm{keV}$ ), revealing the granular (GL) and molecular layer (ML) with typical cell sizes of 5-10 $\mu \mathrm{m}$ (soma), the Purkinje cell layer (PCL), containing the large Purkinje cells (20-40 $\mu \mathrm{m}$ (soma)), and white matter (WM) at cellular detail. (b,c) Magnified view of the regions marked with a rectangle in (a) and (b), respectively. Within the large Purkinje cells, the dendritic tree and the nucleolus as well as to some extent the nucleus are visible. (d) The semi-automatic rendering of a major part of the Purkinje cells reveals their typical flat shape with a highly arbored dendritic tree as well as their parallel arrangement. (e) In a zoom into a specific region of interest recorded in cone-beam geometry (pixel size: $65 \mathrm{~nm}$, energy: $8 \mathrm{keV}$ ) sub-cellular details as the inner structure of the small granule cells can be visualized at high resolution. Scalebars: $200 \mu \mathrm{m}$ (a), $50 \mu \mathrm{m}$ (b) and $20 \mu \mathrm{m}(\mathrm{c}, \mathrm{e})$ 